\title{
CDISC Protocol Terminology
}

National Cancer Institute

\section{Source}

National Cancer Institute. CDISC Protocol Terminology. NCI Thesaurus. Code C132298.

The terminology subset that includes terms relevant to the Clinical Data Interchange

Standards Consortium (CDISC) protocol group. 